\title{
Early childhood respiratory morbidity and antibiotic use in ex-preterm infants: a primary care population-based cohort study
}

\author{
Shin Tan ${ }^{1}$, Lisa Szatkowski², William Moreton ${ }^{1}$, Linda Fiaschi ${ }^{2}$ \\ Tricia McKeever ${ }^{2}$, Jack Gibson ${ }^{2}$ and Don Sharkey $\circledast^{1}$ \\ Affiliations: ${ }^{1}$ Academic Child Health, School of Medicine, University of Nottingham, Nottingham, UK. ${ }^{2}$ Division \\ of Epidemiology and Public Health, School of Medicine, University of Nottingham, Nottingham, UK.
}

Correspondence: Don Sharkey, Academic Child Health, E floor, East Block, University Hospital, Nottingham, NG7 2UH, UK. E-mail: don.sharkeylanottingham.ac.uk

@ERSpublications

This English population-based cohort study in early childhood highlights the significant respiratory morbidity, antibiotic use and increased primary healthcare utilisation in ex-preterm infants, particularly those who were discharged home with oxygen https://bit.ly/2wLeK0g

Cite this article as: Tan S, Szatkowski L, Moreton W, et al. Early childhood respiratory morbidity and antibiotic use in ex-preterm infants: a primary care population-based cohort study. Eur Respir J 2020; 56: 2000202 [https://doi.org/10.1183/13993003.00202-2020].

\section{ABSTRACT}

Background: Globally, bronchopulmonary dysplasia (BPD) continues to increase in preterm infants. Recent studies exploring subsequent early childhood respiratory morbidity have been small or focused on hospital admissions.

Aims: To examine early childhood rates of primary care consultations for respiratory tract infections (RTI), lower respiratory tract infections (LRTI), wheeze and antibiotic prescriptions in ex-preterm and term children. A secondary aim was to examine differences between preterm infants discharged home with or without oxygen.

Methods: Retrospective cohort study using linked electronic primary care and hospital databases of children born between 1997 and 2014. We included 253277 eligible children, with 1666 born preterm at $<32$ weeks' gestation, followed-up from primary care registration to age 5 years. Adjusted incidence rate ratios (aIRRs) were calculated.

Results: Ex-preterm infants had higher rates of morbidity across all respiratory outcomes. After adjusting for confounders, aIRRs for RTI (1.37, 95\% CI 1.33-1.42), LRTI (2.79, 95\% CI 2.59-3.01), wheeze (3.05, 95\% CI 2.64-3.52) and antibiotic prescriptions (1.49, 95\% CI 1.44-1.55) were higher for ex-preterm infants. Ex-preterm infants discharged home on oxygen had significantly greater morbidity across all respiratory diagnoses and antibiotic prescriptions compared to those without home oxygen. The highest rates of respiratory morbidity were observed in children from the most deprived socioeconomic groups. Conclusion: Ex-preterm infants, particularly those with BPD requiring home oxygen, have significant respiratory morbidity and antibiotic prescriptions in early childhood. With the increasing prevalence of $\mathrm{BPD}$, further research should focus on strategies to reduce the burden of respiratory morbidity in these high-risk infants after hospital discharge. 


\section{Introduction}

Survival of preterm, very low birthweight infants has improved with the introduction of antenatal corticosteroids and administration of surfactant, along with better nutrition and ventilation strategies [14]. However, the incidence of bronchopulmonary dysplasia (BPD), a chronic respiratory condition of prematurity, continues to increase and is a major cause of long-term morbidity and mortality in preterm survivors [5-7]. In Europe, the prevalence of BPD in infants born before 32 weeks' gestational age has increased from $23.3 \%$ (2007-2011) to $27.5 \%$ (2012-2015) [8], equating to 28000 affected preterm infants annually based on current birth rates [9]. In the USA, current annual estimates of new cases of BPD range between 10000 and $15000[10,11]$.

BPD is associated with long-term adult respiratory morbidity, including asthma and COPD $[3,12,13]$. In the UK, guidance on post-discharge follow-up for respiratory management in preterm infants with BPD is only provided for those requiring home oxygen, omitting a larger group with less-severe BPD [14]. Moreover, there is a need to explore additional periods in early life that are potentially amenable to alterations of the adverse respiratory trajectory, and hence reduce long-term morbidities. One potential roadblock to achieving these goals is the lack of recent, nationally representative data on early childhood respiratory morbidity in preterm survivors outside of the hospital setting. GREENOUGH et al. [15] studied ex-preterm infants and provided many useful insights into their respiratory health. However, this small study of infants born in 1994-1997 did not provide respiratory diagnoses or antibiotic use, had no term comparison group and follow-up stopped at 2 years of age. A larger, more contemporary study with a term comparator group may provide important information on the respiratory outcomes of these children and identify disparities to be further explored in future research $[16,17]$.

We hypothesised that ex-preterm infants have increased respiratory morbidity in early childhood. The primary aim of our study was to quantify early childhood respiratory morbidity in preterm survivors compared to their term-born counterparts. The secondary aims were to examine differences between preterm infants discharged home with or without oxygen.

\section{Methods}

Data source

This is a retrospective cohort study, using data obtained from the Clinical Practice Research Datalink (CPRD) linked to Hospital Episode Statistics (HES). CPRD is a large, representative national primary care dataset containing anonymised medical records currently encompassing 42 million patient lives from $>1700$ primary care practices in the UK (https://cprd.com/Data). Information is routinely and prospectively recorded for each patient, including patient demographic information, details of consultations, tests and diagnoses and medical prescriptions. HES is a database containing information on all admissions to National Health Service hospitals in England [18]. Clinical information relating to diagnoses and procedures are recorded using codes from the International Classification of Diseases, 10th revision (ICD-10) and UK Office of Population, Census and Surveys (OPCS) Classification of Interventions and Procedures version 4.6, respectively (www.cprd.com/linked-data) [19]. 75\% of active practices contributing to CPRD in England (58\% of all UK practices) are linked with HES, thus providing additional secondary care clinical information for all patients from these practices [20]. We used linked HES data to improve the quality and accuracy of our data, as information such as an infant's birthweight and gestational age are often included in the mother's hospital records. Both databases have been validated with other sources of information and are widely used for epidemiological research [20].

\section{Study population and exposure groups}

We included children born in English hospitals from April 1997 to May 2014 who were registered with a CPRD practice within 6 months of birth and had $\geqslant 3$ months of follow-up data (figure 1 ). They were followed-up from first registration in CPRD until either their fifth birthday, December 31, 2014 (database extraction date) or when they left the general practice. We included all infants with a gestational age of $23+0$ weeks to $43+6$ weeks and with sex-specific birthweights from the 9th to 91 st centiles were included (94.9\%) [21]. This avoided any erroneous entries with birthweight and the majority with abnormal growth patterns, as identification of babies with growth restriction was not possible from this dataset. The distribution of our study population was broadly similar to the population distribution for gestational age and birthweight released by the Office of National Statistics 2010 (supplementary tables S1 and S2), considering that CPRD underrepresents babies born at the extreme of prematurity as it includes only survivors who register with a general practice, whereas ONS data include all registered live births.

For our primary analysis, we identified two cohorts of newborn infants: 1) term infants born $\geqslant 37$ completed weeks of gestation, with birthweights $\geqslant 2500 \mathrm{~g}$ and without prescriptions for home oxygen 


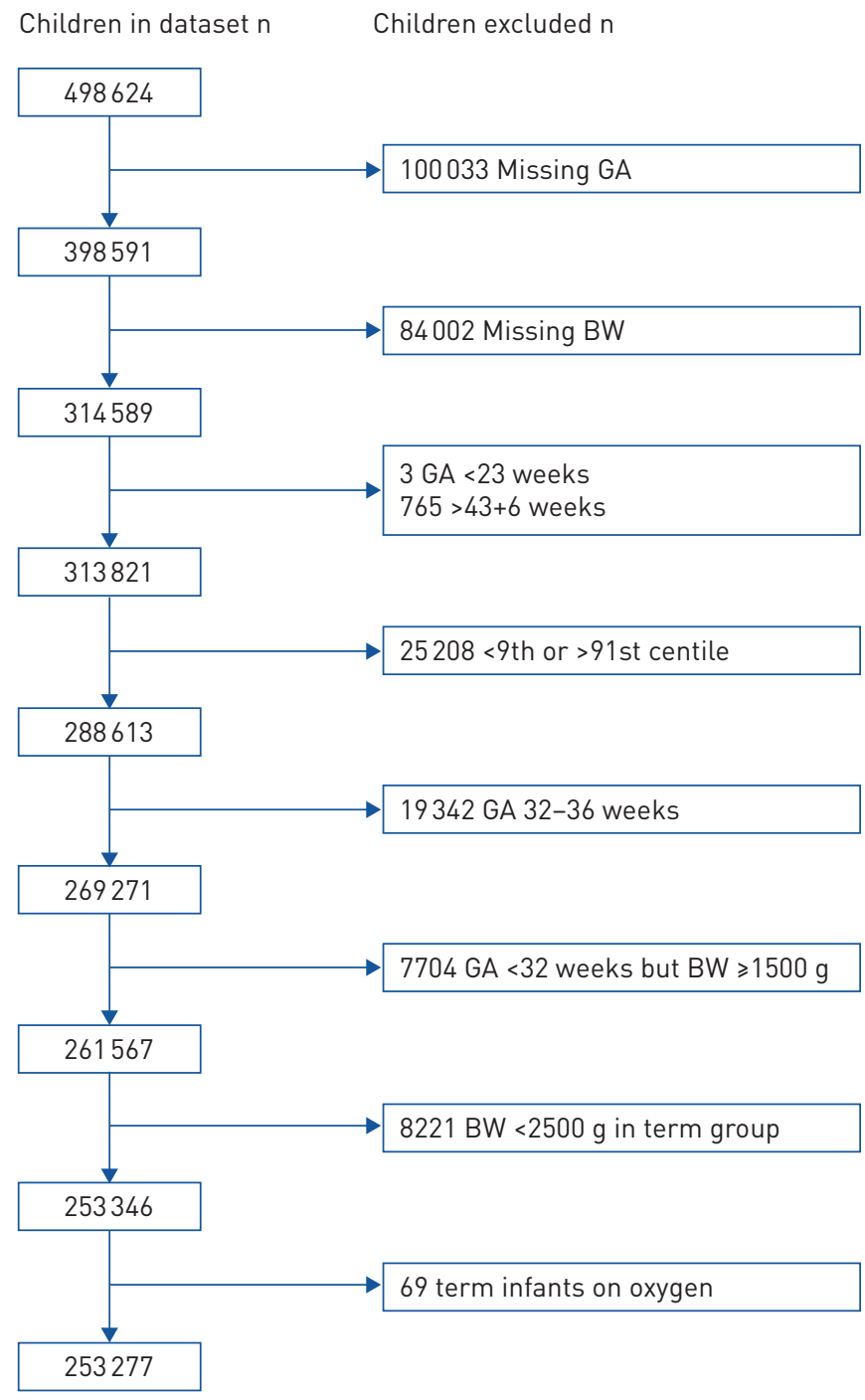

FIGURE 1 Flow diagram illustrating the number of children included and excluded from the cohort at each stage of data cleaning. GA: gestational age; BW: birthweight.

("term"); and 2) preterm infants born before 32 completed weeks of gestation with birthweights $<1500 \mathrm{~g}$ ("preterm"). The preterm group was further divided into two cohorts for secondary subgroup analysis: those with $\left(\mathrm{PT}-\mathrm{O}_{2}\right)$ and without (PT-Air) home oxygen prescriptions in their medical records within 3 months of registration at their general practice.

\section{Outcome variables}

The primary outcomes were defined as 1) rates of primary care consultations for three respiratory conditions: respiratory tract infection (RTI, including both upper and lower RTIs), lower respiratory tract infection specifically (LRTI) and wheeze; and 2) rates of antibiotic prescriptions.

Episodes of respiratory outcomes were identified using Read medical codes recorded in primary care records (available from the authors on request) [22, 23]. For example, an RTI was defined based on a recorded clinical diagnosis of a relevant infection (e.g. acute respiratory infection, acute pharyngitis) or an infection-related symptom (e.g. cough with fever, sore throat) during the follow-up period. As previously, we defined recovery as the 15th day after the initial diagnosis of infection [22, 23] with any subsequent diagnosis considered to be the same infection episode and the recovery date was extended to the eighth day of this recording (if later than original recovery date), to ensure each episode lasted $\geqslant 14$ days.

Antibiotic prescriptions were identified using Multilex drug codes (drug codes available from the authors on request). We included antibiotics detailed as suitable for treating respiratory infections, as defined by the British National Formulary for Children (BNFC) (https://bnfc.nice.org.uk) in the following classes: penicillins, cephalosporins and other $\beta$-lactams, macrolides, and sulphonamides and trimethoprim. 
Other covariables

Data were extracted on descriptive characteristics and covariables including sex, socioeconomic status measured using the Index of Multiple Deprivation (IMD) 2010 [24, 25], and presence of major congenital anomalies based on the European Surveillance of Congenital Anomalies (EUROCAT; https:// eu-rd-platform.jrc.ec.europa.eu/eurocat_en) definition. Outcomes were quantified based on yearly age bands from birth to 5 years.

\section{Statistical analyses}

Data management and analyses were undertaken using Stata (version 14; StataCorp, College Station, TX, USA). The number of episodes of respiratory infection, antibiotic prescriptions and crude incidence rate per 10 person-years with 95\% confidence intervals were calculated both overall and separately for each covariable. We used negative binomial regression to generate incidence rate ratios (IRR) comparing preterm to term infants with adjustment for potential confounders, including yearly age bands, sex, IMD 2010 quintiles and presence of congenital anomalies.

To examine the differences in outcomes between PT-Air and PT- $\mathrm{O}_{2}$ infants, we repeated the above steps as further subgroup analyses and compared these results to their term counterparts.

In addition, we conducted the analyses over two epochs to assess changes in respiratory morbidity over time. We divided the study period into two equal 8-year epochs: epoch 1 (1997-2005) and epoch 2 (20062014).

\section{Ethical approval}

The providers of the CPRD data received ethical approval from a National Research Ethics Service Committee (NRES) for data collection and subsequent observational research using anonymised data; individual studies using CPRD data do not require further separate ethical approval. The protocol for this project was approved by the CPRD independent study advisory committee (reference:16_189R).

\section{Results}

Baseline characteristics

We identified 253277 eligible children: 251611 children in the term group and 1666 children in the preterm group (table 1). More children in the preterm group (44.9\%) were from more deprived socioeconomic backgrounds (IMD 2010 fourth and fifth quintiles) compared to those in the term group $(39.3 \%)$.

\section{Respiratory tract infections}

The overall crude incidence rate for consultations for RTI in the term and preterm groups were 7.17 (95\% CI 7.15-7.19) and 9.69 (95\% CI 9.42-9.96), respectively. The adjusted (a)IRR for the preterm group was

\begin{tabular}{|c|c|c|c|}
\hline & $\begin{aligned} \text { Term } & \geqslant 37 / 40 \text { weeks and } \\
& \geqslant 2500 \mathrm{~g}\end{aligned}$ & $\begin{aligned} \text { Preterm } & <32 / 40 \text { weeks and } \\
& <1500 \mathrm{~g}\end{aligned}$ & p-value \\
\hline Subjects & 251611 & 1666 & \\
\hline Male & $130220(51.8)$ & 815 (48.9) & 0.021 \\
\hline Gestational age at birth weeks & $40(39-41)$ & $28(27-30)$ & $<0.001$ \\
\hline Birthweight $\mathbf{g}$ & 3459 (3180-3745) & $1168(966-1330)$ & $<0.001$ \\
\hline \multicolumn{4}{|l|}{ IMD 2010 quintile } \\
\hline 1 (least deprived) & 54154 (21.5) & $280(16.8)$ & $<0.001$ \\
\hline 2 & $51410(20.4)$ & 330 (19.8) & \\
\hline 3 & $46687(18.6)$ & 305 (18.3) & \\
\hline 4 & 49899 (19.8) & $364(21.9)$ & \\
\hline 5 (most deprived) & 49071 (19.5) & $383(23.0)$ & \\
\hline Missing & $390(0.2)$ & $4(0.2)$ & \\
\hline $\begin{array}{l}\text { Age at the beginning of } \\
\text { follow-up days }\end{array}$ & $25(14-43)$ & $49(29-75)$ & $<0.001$ \\
\hline Length of follow-up months & $48(24-60)$ & $49(23-60)$ & $<0.001$ \\
\hline Congenital anomalies & $6158(2.5)$ & $333(20)$ & $<0.001$ \\
\hline
\end{tabular}

Data are presented as $\mathrm{n}, \mathrm{n}(\%)$ or median (interquartile range), unless otherwise stated. IMD: Index of Multiple Deprivation. 
TABLE 2 Overall incidence rates and incidence rate ratios (IRRs) (unadjusted and adjusted) for respiratory outcomes in the first 5 years of life in term and preterm cohorts

\begin{tabular}{|c|c|c|c|c|c|c|}
\hline & \multicolumn{2}{|r|}{ Term } & \multicolumn{2}{|r|}{ Preterm } & \multirow[t]{2}{*}{ Unadjusted IRR (95\% CI) } & \multirow[t]{2}{*}{ Adjusted IRR ${ }^{\#}(95 \%$ CI) } \\
\hline & Events $\mathrm{n}$ & Rate $(95 \% \mathrm{CI})$ & Events $\mathrm{n}$ & Rate $(95 \% \mathrm{CI})$ & & \\
\hline RTI & 451438 & $7.17(7.15-7.19)$ & 4940 & $9.69(9.42-9.96)$ & $1.35^{* * *}(1.31-1.39)$ & $1.37 * * *(1.33-1.42)$ \\
\hline Wheeze & 32529 & $0.52(0.51-0.52)$ & 608 & $1.19(1.10-1.29)$ & $2.31^{* * *}(2.13-2.50)$ & $3.05^{* * *}(2.64-3.52)$ \\
\hline Antibiotic prescriptions & 711743 & $8.40(8.38-8.42)$ & 6971 & $12.67(12.38-12.97)$ & $1.51 * * *(1.47-1.54)$ & $1.49 * * *(1.44-1.55)$ \\
\hline
\end{tabular}

All rates are described as per 10 person-years. RTI: respiratory tract infection; LRTI: lower respiratory tract infection. \#: adjusted for age, sex, Index of Multiple Deprivation 2010 quintile, congenital anomalies. ${ }^{* * *}$ : $p<0.001$.

1.37 (95\% CI 1.33-1.42, $\mathrm{p}<0.001$ ) after adjustment for confounding factors (table 2, figure 2). Both term and preterm groups had the highest incidence rate of consultations for RTI within their first year of life, which gradually decreased over the first 5 years of childhood. However, the incidence rate in the preterm group was significantly higher than the term group in all age groups $(p<0.001)$. The highest incidence rate of consultations for RTIs were found in the most socioeconomically deprived group (supplementary table S3). aIRRs were also increased for children in the preterm group compared to the term group (supplementary table S3).

\section{Lower respiratory tract infections}

Children in the preterm group had a significantly higher overall incidence rate for consultations for LRTI at 2.74 (95\% CI $2.60-2.89)$ compared to 1.14 (95\% CI 1.13-1.15) in the term group. After adjustments, the IRR for consultations for LRTI in the preterm group in the first 5 years of life was 2.79 (95\% CI 2.59-3.01, $\mathrm{p}<0.001$ ) (table 2). The rates of consultations for LRTI in the preterm group were consistently higher than those in the term group across all age groups, peaking in the second year of life before declining. Consultations for LRTIs were the highest in the most deprived populations for both term and preterm infants. As seen with consultations for RTI, all aIRRs were increased in the preterm group (supplementary table S4).
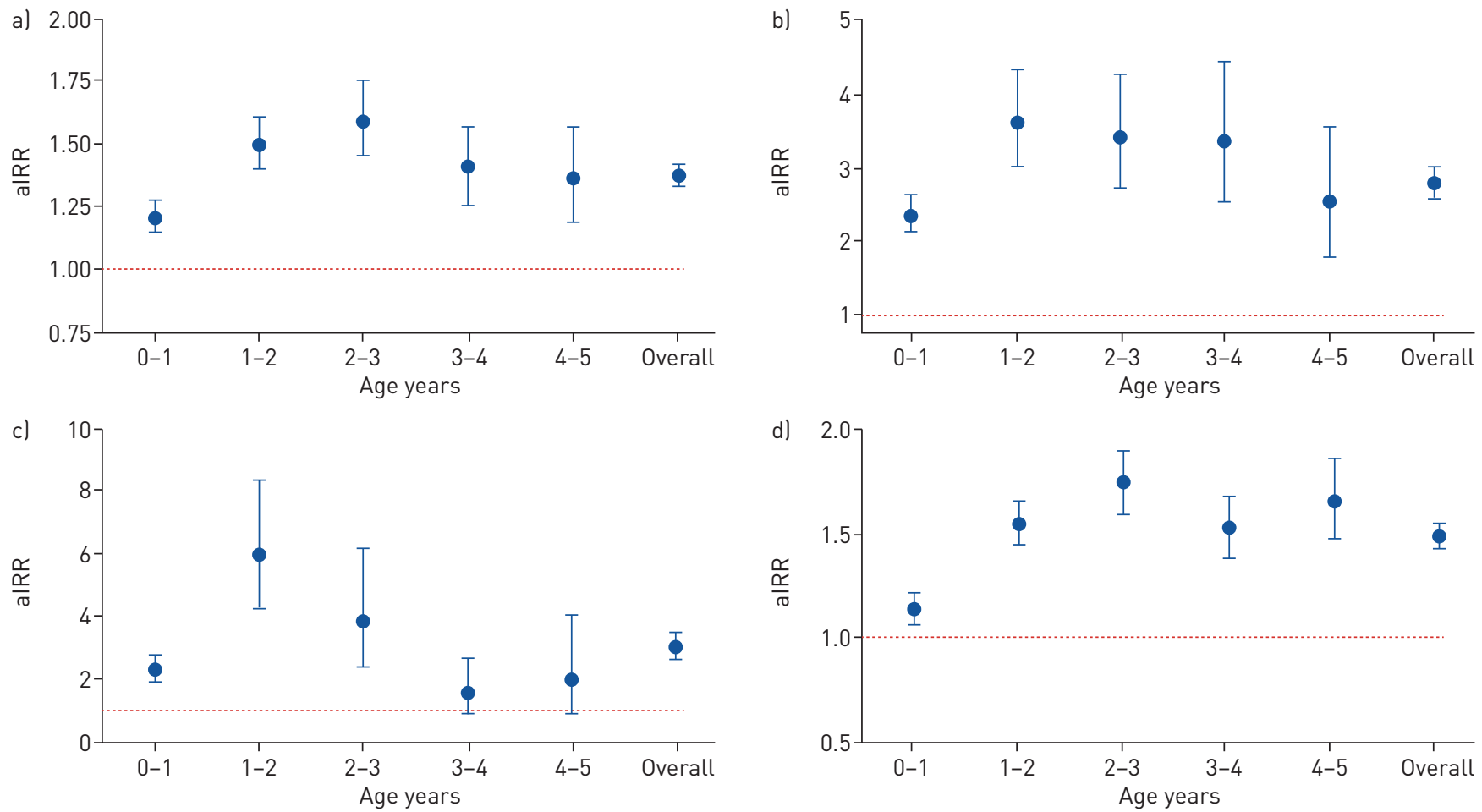

FIGURE 2 Overall adjusted incidence rate ratios (alRRs) for respiratory outcomes comparing term and preterm cohorts over 5 years of follow-up. a) Respiratory tract infection; b) lower respiratory tract infection; c) wheeze; d) antibiotic prescriptions. 
Wheeze

The overall incidence rate of consultations for wheeze in the preterm cohort was more than double that in the term cohort (table 2), with an aIRR of 3.05 (95\% CI 2.64-3.52, p<0.001). Wheeze was more likely to be diagnosed in the first year of life for term infants, but peaked in the second year of life for preterm infants. Rates of consultations for wheeze were significantly higher in the preterm population throughout all age groups and deprivation quintiles (supplementary table S5).

\section{Antibiotic prescriptions}

Ex-preterm infants had significantly higher rates of antibiotic prescriptions compared to their term counterparts with overall incidence rate of 12.67 (95\% CI 12.38-12.97) and 8.40 (95\% CI 8.38-8.42), respectively (table 2, figure 3). The aIRR for the preterm group was 1.49 (95\% CI 1.44-1.55, $\mathrm{p}<0.001$ ). Children in both cohorts had the highest rate of antibiotic prescriptions in their second year of life before gradually decreasing. There were no significant differences in prescribing based on socioeconomic background (supplementary table S6). The major classes of antibiotics prescribed by group are shown in supplementary table S7.

\section{Subgroup analyses}

Infants in the PT- $\mathrm{O}_{2}$ group were more likely to have lower gestational ages compared to those in the PT-Air group (table 3). There was a significant increase in morbidity across all three respiratory outcomes and antibiotic prescriptions in the PT- $\mathrm{O}_{2}$ group compared to the PT-Air group (table 4, supplementary tables S8 and S9). Compared with the PT-Air group, the $\mathrm{PT}-\mathrm{O}_{2}$ had higher aIRRs for consultations for RTI (1.21, 95\% CI 1.12-1.30; p<0.001), LRTI (1.74, 95\% CI 1.50-2.03; p<0.001) and antibiotic prescriptions $(1.39,95 \%$ CI $1.28-1.50 ; \mathrm{p}<0.001)$. In addition, the $\mathrm{PT}-\mathrm{O}_{2}$ group had higher incidence rates for consultations for wheeze; however, this was not statistically significant following adjustment. Rates of consultations for RTI and LRTI observed in the $\mathrm{PT}-\mathrm{O}_{2}$ group were consistently higher than those in the PT-Air group across all age groups (figure 3). In particular, the rate of consultations for LRTI seen in the
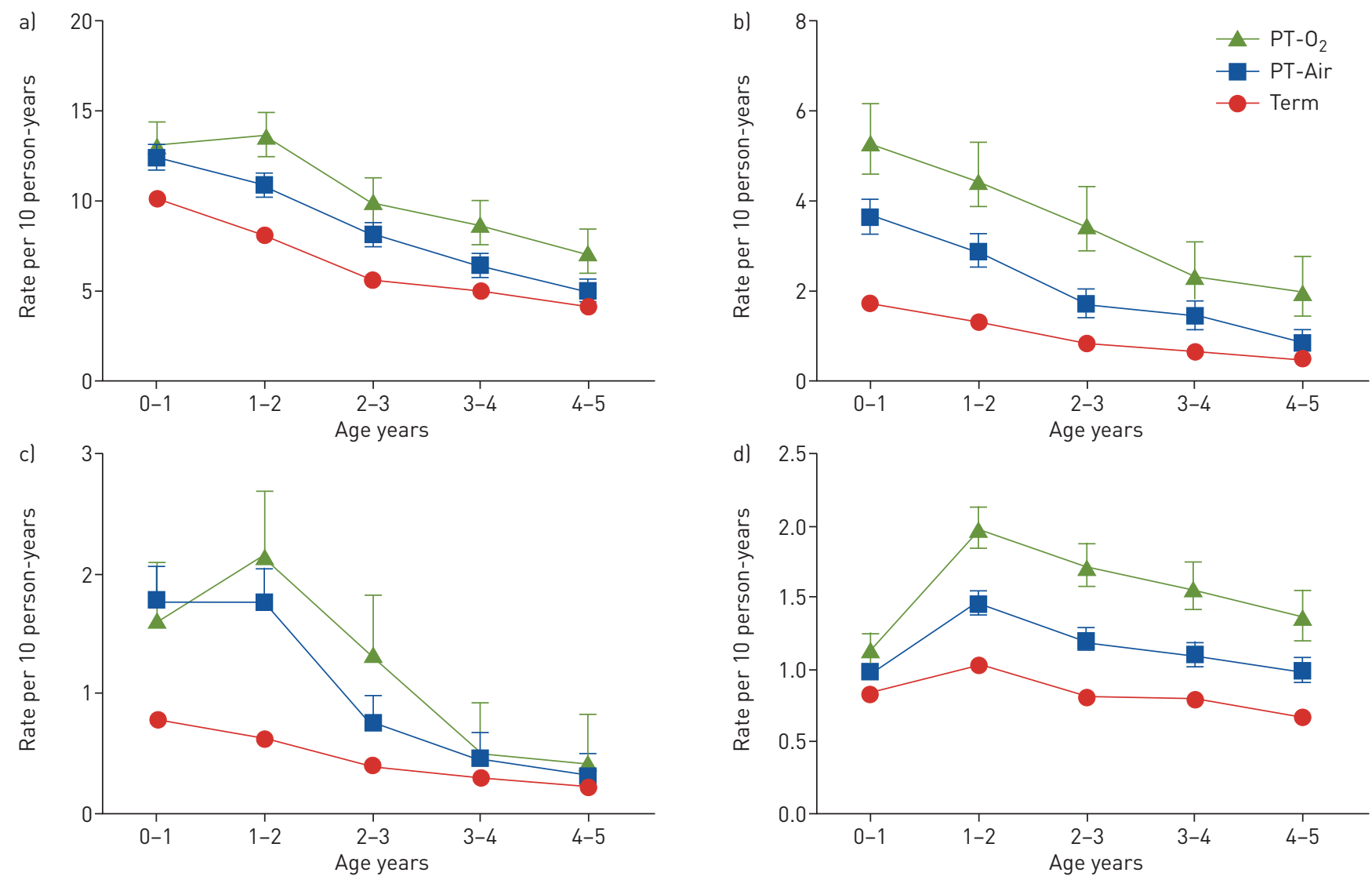

FIGURE 3 Crude incidence rates of respiratory outcomes al respiratory tract infection; b) lower respiratory tract infection; c) wheeze) and d) antibiotic prescriptions across all three cohorts over the first 5 years of childhood. PT- $\mathrm{O}_{2}$ : preterm infants with home oxygen prescriptions in their medical records within 3 months of registration at their general practice; PT-Air: preterm infants without home oxygen prescriptions in their medical records within 3 months of registration at their general practice. 


\begin{tabular}{|c|c|c|}
\hline & PT-Air & PT- $\mathrm{O}_{2}$ \\
\hline Subjects & 1217 & 449 \\
\hline Male & 575 (47.2) & $240(53.5)$ \\
\hline Gestational age at birth weeks & $29(28-30)$ & $26(25-28)$ \\
\hline Birthweight g & $1245(1077-1360)$ & 955 (794-1095) \\
\hline \multicolumn{3}{|l|}{ IMD 2010 quintile } \\
\hline 1 (least deprived) & 207 (17.0) & 73 (16.3) \\
\hline 2 & 235 (19.3) & $95(21.2)$ \\
\hline 3 & $231(19.0)$ & $74(16.5)$ \\
\hline 4 & 259 (21.3) & $105(23.4)$ \\
\hline 5 (most deprived) & $284(23.3)$ & $99(22.0)$ \\
\hline Missing & $1(0.1)$ & $3(0.6)$ \\
\hline Congenital anomalies & $175(14.4)$ & $158(35.2)$ \\
\hline
\end{tabular}

Data are presented as $\mathrm{n}, \mathrm{n}(\%)$ or median (interquartile range). IMD: Index of Multiple Deprivation.

PT- $\mathrm{O}_{2}$ group was three to four times higher than that seen in the term group across all 5 years of early childhood.

\section{Epoch analyses}

The study cohort was divided into two 8-year epochs: epoch 1 (1997-2005) included 87556 children and epoch 2 (2006-2014) included 165721 children (supplementary table S10). Overall, there were no substantial differences in the aIRRs for consultations for RTI, LRTI and wheeze between two epochs; the 95\% confidence intervals for the two epochs for these outcomes overlap (table 5). The aIRR for antibiotic prescriptions decreased from 1.58 (95\% CI 1.50-1.67) to 1.41 (95\% CI 1.34-1.48).

\section{Discussion}

This study, based on a large nationally representative sample of young children in England, highlights a vulnerable cohort of ex-preterm children with significant respiratory morbidity in early childhood compared to children born at term. Ex-preterm infants had higher rates of primary care diagnosed respiratory infections, especially LRTI, which are more likely to result in the need for antibiotics [26]. For ex-preterm infants discharged on home oxygen, the rates of consultations for LRTI and antibiotic prescriptions are even greater and remain high throughout childhood and never fall below that of term babies across all years.

Prematurity and low birthweight were associated with a persistent increase in respiratory morbidity in early childhood. This appeared magnified for the infants in the $\mathrm{PT}-\mathrm{O}_{2}$ cohort, who had significantly worse respiratory outcomes compared to those in the PT-Air cohort. The rates of consultations for RTI seen in our study are higher than those previously reported [27]; however, those were reported from hospital admissions for respiratory conditions. The highest rates of infection-related consultations were mostly observed in the first year of life when innate immune responses predominate, in keeping with other large

TABLE 4 Overall incidence rates and incidence rate ratios (IRRs) (unadjusted and adjusted) for respiratory outcomes in the first 5 years of life in preterm children without (PT-Air) and with (PT- $\mathrm{O}_{2}$ ) home oxygen

\begin{tabular}{|c|c|c|c|c|c|c|}
\hline & \multicolumn{2}{|r|}{ PT-Air } & \multicolumn{2}{|r|}{ PT- $\mathrm{O}_{2}$} & \multirow[t]{2}{*}{ Unadjusted IRR (95\% CI) } & \multirow[t]{2}{*}{ Adjusted IRR ${ }^{\#}(95 \%$ CI) } \\
\hline & Events $\mathrm{n}$ & Rate $(95 \% \mathrm{CI})$ & Events n & Rate $(95 \% \mathrm{CI})$ & & \\
\hline RTI & 3431 & $9.16(8.85-9.47)$ & 1509 & $11.16(10.62-11.74)$ & $1.22^{* * *}(1.15-1.30)$ & $1.21 * * *(1.12-1.30)$ \\
\hline Wheeze & 426 & $1.14(1.03-1.25)$ & 182 & $1.35(1.16-1.56)$ & $1.18 *(0.99-1.41)$ & $1.10(0.86-1.42)$ \\
\hline Antibiotic prescriptions & 4768 & 11.63 (11.31-11.97) & 2203 & $15.71(15.06-16.38)$ & $1.35^{* * *}(1.28-1.42)$ & $1.39 * * *(1.28-1.50)$ \\
\hline
\end{tabular}

All rates are described as per 10 person-years. RTI: respiratory tract infection; LRTI: lower respiratory tract infection. ${ }^{\text {: }}$ : adjusted for age, sex, Index of Multiple Deprivation 2010 quintile, congenital anomalies. ${ }^{*}$ : $p<0.05 ; * * *$ : $p<0.001$. 
TABLE 5 Epoch analyses showing unadjusted and adjusted incidence rate ratios (IRRs) between term and preterm cohorts

\begin{tabular}{|c|c|c|c|c|}
\hline & \multicolumn{2}{|c|}{ Epoch 1 (1997-2005) } & \multicolumn{2}{|c|}{ Epoch 2 (2006-2014) } \\
\hline & Unadjusted IRR (95\% CI) & Adjusted IRR ${ }^{\#}(95 \% \mathrm{CI})$ & Unadjusted IRR (95\% CI) & Adjusted IRR $(95 \% \mathrm{Cl})$ \\
\hline RTI & $1.34^{* * *}(1.28-1.40)$ & $1.36 * * *(1.29-1.43)$ & $1.36^{* * *}(1.31-1.42)$ & $1.37 * * *(1.30-1.43)$ \\
\hline Wheeze & $1.95^{* * *}(1.71-2.22)$ & $2.48 * * *(1.98-3.10)$ & $2.60 * * *(2.34-2.88)$ & $3.36 * * *(2.79-4.05)$ \\
\hline Antibiotic prescriptions & $1.52^{* * *}(1.47-1.58)$ & $1.58 * * *(1.50-1.67)$ & $1.49 * * *(1.44-1.54)$ & $1.41 * * *(1.34-1.48)$ \\
\hline
\end{tabular}

RTI: respiratory tract infection; LRTI: lower respiratory tract infection. \#: adjusted for age, sex, Index of Multiple Deprivation 2010 quintile, congenital abnormalities. ${ }^{* * *}: \mathrm{p}<0.001$.

hospital-based epidemiological studies published previously [28]. These infective episodes were more common in both the more deprived term and preterm groups, confirming the adverse impact of social deprivation on child respiratory health.

Our study highlights the significant respiratory burden presenting to primary care in ex-preterm infants, especially those discharged on home oxygen who are more likely to have severe BPD. There is a paucity of data in this population and the rates of presentation to primary care. A number of small studies have focused on the respiratory function of these ex-preterm infants rather than the clinical burden in the community. The long-term respiratory function of ex-preterm infants has been reported previously whereby childhood impairment of lung function is a significant predictor of abnormal longitudinal patterns of lung function growth and decline into adulthood [29]. GougH et al. [12] and YANG et al. [30] have reported persistent adult respiratory morbidity in ex-preterm infants with BPD, including more significant impairment of lung function and increased respiratory symptoms.

As extreme preterm survival and BPD continue to rise, along with the associated lifelong respiratory morbidity, it is extremely important to explore key periods in early life amenable to interventions to improve long-term respiratory health. It is unclear who is best placed to manage and support the respiratory health of these children following discharge from the neonatal unit. The high rates of consultations for LRTI and antibiotic prescriptions in the PT- $\mathrm{O}_{2}$ group would suggest that these children may benefit from expert paediatric respiratory follow-up. In our study we are unable to ascertain which children had such follow-up, but a previous study suggests $<20 \%$ of extremely preterm children with moderate/severe BPD have paediatric follow-up at 6 years of age [31]. In the UK, British Thoracic Society (BTS) guidelines for home oxygen recommend that infants should have hospital follow-up either at a tertiary centre or district general hospital if the general paediatrician has experience of home oxygen [14]. As respiratory physicians have the specialty expertise in managing and optimising lung health, it is currently unclear whether targeted paediatric respiratory follow-up would reduce respiratory morbidity in this cohort of children; an approach the Prematurity and Respiratory Outcomes Program (PROP) in the US has identified with the need for integrated, multidisciplinary research and clinical teams [32].

Our study aimed to report on primary care consultations where general practitioners (GPs) make the clinical diagnosis of infection and decide if antibiotics are indicated. Higher levels of anxiety among parents with ex-preterm infants may lead to increased health-seeking behaviour [33]. Equally, GPs may be more anxious about this cohort of children given their clinical history or comorbidities and so have lower thresholds to prescribe antibiotics for such infections. These could contribute to the high rates of antibiotic prescriptions seen prescribed to both preterm cohorts in our study. Antimicrobial resistance is a serious global concern at a time when prescribing antibiotics is at an all-time high [34], therefore additional parental education and support for GPs could help improve antimicrobial stewardship in these children.

Our study is the largest to date, from a robust and well-validated data source [20], quantifying a previously unrecorded burden of primary healthcare utilisation and respiratory morbidity in a recent ex-preterm population. Previous studies in this population have focused on hospital admissions only or did not allow comparison between preterm infants discharged with or without home oxygen and term infants in the same population $[15,27]$.

There appears to be no significant differences over the study time period as observed by the epoch analysis, with consultations for infection and wheeze rate differences between term and preterm unchanged. Antibiotic prescription rates remain higher for the preterm population, although the difference appears slightly lower in recent years. The reasons for this are unclear, especially with similar rates of 
infection being diagnosed, but are perhaps related to better antibiotic stewardship, smaller numbers in the preterm groups as a consequence of epoch division or other mechanisms not measured in this study.

There are a number of limitations of this study including its retrospective nature, although data were collected prospectively. The lack of paired respiratory diagnosis and antibiotic prescriptions makes it difficult to directly link these episodes, although we only included antibiotics which may be used for respiratory infections. Some of these drugs could be used for other infections; for example, trimethoprim is used for urinary tract infections. The database analysis does not allow us to classify which preterm children had a diagnosis of BPD. We therefore used home oxygen as a proxy for more severe respiratory disease, although this requirement can be related to morbidities other than BPD, or other factors not modelled, such as gestational age. In addition, prospective data on which specialists undertake outpatient follow-up of the preterm group would help understanding of any impact on respiratory morbidity. The present study focused on primary care consultations which have not been studied in such detail. Extension of this study to include hospital attendances was beyond the scope of the study, but could be useful supporting the morbidity observed as previously reported [27, 35].

Finally, we had no data on children's exposure to cigarette smoke, either in utero or passive exposure during childhood. Recording of smoking status of pregnant women in primary care data is incomplete [36] and data recorded may not accurately reflect women's true smoking status. Nevertheless, we acknowledge the impact of maternal smoking on health and lung function, including the increasing prevalence of childhood respiratory infections and wheeze [32]. As smoking is more prevalent in lower socioeconomic populations this could contribute to some of the additional morbidity observed in the preterm groups

\section{Conclusions}

Our findings highlight significant respiratory morbidity in ex-preterm infants, especially those discharged home on oxygen and likely to have more severe BPD, in the first 5 years of life. With increasing survival and BPD in this population there could be an increased health resources implication for both hospital physicians and GPs. Additional research is needed on prevention strategies to minimise respiratory morbidity and improve long-term lung function for these high-risk children. Key to this is the identification of core respiratory outcomes most important for both clinicians and parents, and for targeted interventions to ameliorate these. Longer-term respiratory follow-up is essential as more of these vulnerable infants survive to reduce the burden for children, their families and healthcare services. Who is best placed to undertake long-term respiratory follow-up remains to be established.

Author contributions: D. Sharkey and L. Szatkowski designed the study. L. Szatkowski and L. Fiaschi provided data and undertook data linkage. S. Tan built the final dataset for the study. S. Tan and W. Moreton undertook the statistical analyses under supervision of L. Fiaschi, T. McKeever and J. Gibson. All authors interpreted the results. S. Tan drafted the manuscript, and all other authors contributed revisions.

Conflict of interest: None declared.

\section{References}

1 Kinsella JP, Greenough A, Abman SH. Bronchopulmonary dysplasia. Lancet 2006; 367: 1421-1431.

2 Siffel C, Kistler KD, Lewis JFM, et al. Global incidence of bronchopulmonary dysplasia among extremely preterm infants: a systematic literature review. J Matern Fetal Neonatal Med 2019: 1-11.

3 Duijts L, van Meel ER, Moschino L, et al. European Respiratory Society guideline on long term management of children with bronchopulmonary dysplasia. Eur Respir J 2019; 55: 1900788.

4 Owen LS, Cheong JLY, Davis PG. Bronchopulmonary dysplasia as a trial endpoint: time for re-evaluation? Lancet Child Adolesc Health 2019; 3: 842-844.

5 Eber E, Zach MS. Long term sequelae of bronchopulmonary dysplasia (chronic lung disease of infancy). Thorax 2001; 56: 317-323.

6 Ehrenkranz RA, Walsh MC, Vohr BR, et al. Validation of the National Institutes of Health consensus definition of bronchopulmonary dysplasia. Pediatrics 2005; 116: 1353-1360.

7 Stoll BJ, Hansen NI, Bell EF, et al. Trends in care practices, morbidity, and mortality of extremely preterm neonates, 1993-2012. JAMA 2015; 314: 1039-1051.

8 Lui K, Lee SK, Kusuda S, et al. Trends in outcomes for neonates born very preterm and very low birthweight in 11 high-income countries. J Pediatr 2019; 215: 32-40.

9 Chawanpaiboon S, Vogel JP, Moller A-B, et al. Global, regional, and national estimates of levels of preterm birth in 2014: a systematic review and modelling analysis. Lancet Glob Health 2019; 7: e37-e46.

10 Islam JY, Keller RL, Aschner JL, et al. Understanding the short- and long-term respiratory outcomes of prematurity and bronchopulmonary dysplasia. Am J Respir Crit Care Med 2015; 192: 134-156.

11 Jensen EA, Schmidt B. Epidemiology of bronchopulmonary dysplasia. Birth Defects Res A Clin Mol Teratol 2014; 100: 145-157.

12 Gough A, Linden M, Spence D, et al. Impaired lung function and health status in adult survivors of bronchopulmonary dysplasia. Eur Respir J 2014; 43: 808-816. 
13 Álvarez-Fuente $\mathrm{M}$, Arruza L, Muro $\mathrm{M}$, et al. The economic impact of prematurity and bronchopulmonary dysplasia. Eur J Pediatr 2017; 176: 1587-1593.

14 Balfour-Lynn IM, Field DJ, Gringras P, et al. BTS guidelines for home oxygen in children. Thorax 2009; 64: Suppl. 2, ii1-ii26.

15 Greenough A, Alexander J, Burgess S, et al. Home oxygen status and rehospitalisation and primary care requirements of infants with chronic lung disease. Arch Dis Child 2002; 86: 40-43.

16 Vrijlandt EJLE, Kerstjens JM, Duiverman EJ, et al. Moderately preterm children have more respiratory problems during their first 5 years of life than children born full term. Am J Respir Crit Care Med 2013; 187: 1234-1240.

17 Montgomery S, Bahmanyar S, Brus O, et al. Respiratory infections in preterm infants and subsequent asthma: a cohort study. BMJ Open 2013; 3: e004034.

18 NHS Digital. Hospital Episode Statistics (HES). https://digital.nhs.uk/data-and-information/data-tools-andservices/data-services/hospital-episode-statistics Date last accessed: May 18, 2018. Date last updated: March 26, 2019.

19 Padmanabhan S, Carty L, Cameron E, et al. Approach to record linkage of primary care data from Clinical Practice Research Datalink to other health-related patient data: overview and implications. Eur J Epidemiol 2019; 34: 91-99.

20 Herrett E, Gallagher AM, Bhaskaran K, et al. Data resource profile: Clinical Practice Research Datalink (CPRD). Int J Epidemiol 2015; 44: 827-836.

21 Freeman JV, Cole TJ, Chinn S, et al. Cross sectional stature and weight reference curves for the UK, 1990. Arch Dis Child 1995; 73: 17-24.

22 Ban L, Gibson JE, West J, et al. Association between perinatal depression in mothers and the risk of childhood infections in offspring: a population-based cohort study. BMC Public Health 2010; 10: 799.

23 Bannister J, Szatkowski L, Sharkey D, et al. Early life incidence of gastrointestinal and respiratory infections in children with gastroschisis: a cohort study. J Pediatr Gastroenterol Nutr 2018; 67: 580-585.

24 Ministry of Housing, Communities \& Local Government. English Indices of Deprivation 2010. www.gov.uk/ government/statistics/english-indices-of-deprivation-2010 Date last accessed: November 20, 2019.

25 Oxford Consultants for Social Inclusion (OCSI). Headline results from the Indices of Deprivation 2010. https:// ocsi.uk/2011/03/24/headline-results-from-the-indices-of-deprivation-2010/ Date last accessed: November 20, 2019.

26 National Institute for Health and Care Excellence (NICE). Respiratory tract infections (self-limiting): prescribing antibiotics. www.nice.org.uk/guidance/cg69. Date last accessed: January 23, 2020. Date last updated: July 23, 2008.

27 Paranjothy S, Dunstan F, Watkins WJ, et al. Gestational age, birthweight, and risk of respiratory hospital admission in childhood. Pediatrics 2013; 132: e1562-e1569.

28 Miller JE, Hammond GC, Strunk T, et al. Association of gestational age and growth measures at birth with infection-related admissions to hospital throughout childhood: a population-based, data-linkage study from Western Australia. Lancet Infect Dis 2016; 16: 952-961.

29 McGeachie MJ, Yates KP, Zhou X, et al. Patterns of growth and decline in lung function in persistent childhood asthma. N Engl J Med 2016; 374: 1842-1852.

30 Yang J, Kingsford RA, Horwood J, et al. Lung function of adults born at very low birthweight. Pediatrics 2020; 145: e20192359.

31 Hennessy EM, Bracewell MA, Wood N, et al. Respiratory health in pre-school and school age children following extremely preterm birth. Arch Dis Child 2008; 93: 1037-1043.

32 Higgins RD, Jobe AH, Koso-Thomas $\mathrm{M}$, et al. Bronchopulmonary dysplasia: executive summary of a workshop. J Pediatr 2018; 197: 300-308.

33 Holditch-Davis D, Santos H, Levy J, et al. Patterns of psychological distress in mothers of preterm infants. Infant Behav Dev 2015; 41: 154-163.

34 Palin V, Mölter A, Belmonte M, et al. Antibiotic prescribing for common infections in UK general practice: variability and drivers. J Antimicrob Chemother 2019; 74: 2440-2450.

35 Haataja P, Korhonen P, Ojala R, et al. Hospital admissions for lower respiratory tract infections in children born moderately/late preterm. Pediatr Pulmonol 2018; 53: 209-217.

36 Dhalwani NN, Tata LJ, Coleman T, et al. Completeness of maternal smoking status recording during pregnancy in United Kingdom primary care data. PLoS One 2013; 8: e72218. 